\title{
IMPACT of PCSK9 inhibition on clinical outcome in patients during the inflammatory stage of the SARS-COV-2 infection: Rationale and protocol of the IMPACT-SIRIO 5 study
}

\author{
Jacek Kubica ${ }^{1}$, Przemysław Podhajski ${ }^{1}$, Przemysław Magielski ${ }^{1}$, Aldona Kubica ${ }^{2}$, \\ Piotr Adamski ${ }^{1}$, Roman Junik ${ }^{3}$, Jarosław Pinkas ${ }^{4}$, Eliano P. Navarese ${ }^{1}$ \\ ${ }^{1}$ Department of Cardiology and Internal Medicine, Collegium Medicum, \\ Nicolaus Copernicus University, Bydgoszcz, Poland \\ ${ }^{2}$ Department of Health Promotion, Nicolaus Copernicus University, \\ Collegium Medicum in Bydgoszcz, Poland \\ ${ }^{3}$ Department of Endocrinology and Diabetology, Collegium Medicum in Bydgoszcz, \\ Nicolaus Copernicus University in Torun, Poland \\ ${ }^{4}$ Center of Postgraduate Medical Education, School of Public Health, Warsaw, Poland
}

\section{Introduction}

Coronavirus disease 2019 (COVID-19) infection is characterized by distinct stages: the first one with dominant replication of the virus, and the second one with dominant immunological inflammatory response of the host [1]. COVID-19 can evolve into a systemic severe inflammatory response characterized by cytokine storm and acute respiratory distress syndrome. Cytokine storm refers to a set of clinical conditions caused by excessive immune reactions and has been recognized as a leading cause of severe COVID-19, multiple organ dysfunction syndrome and adverse clinical outcomes [2-6]. Various laboratory markers have been linked with COVID-19-related excessive inflammatory response caused by cytokine release syndrome. Among cytokines, interleukin (IL)-6, IL-8, and tumor necrosis factor alpha (TNF- $\alpha$ ) are regarded as the most relevant triggers of the hyperinflammatory reaction during COVID-19 [7-9]. Furthermore, microvascular and macrovascular thrombosis are observed complications in COVID-19 which in turn may be the target of therapeutic strategies to significant influence disease-related sequelae and mortality [5-7].

Proprotein convertase subtilisin/kexin type 9 (PCSK9) is an enzyme that plays a crucial role in the homeostasis of low-density lipoprotein (LDL) receptors (LDLR), however, it also promotes inflammatory response [10]. Experimental and clinical data suggest that in addition to improvement in lipid profile and clinical outcomes in patients with cardiovascular diseases, PCSK9 inhibitors also exert an anti-inflammatory effect that might be related to interference on the IL-6 pathway [10-15].

Herein, is a discussion of the rationale for the use of PCSK9 inhibitors in the treatment during the hyperinflammatory stage of COVID-19 and present a design of the ongoing study in testing this hypothesis (NCT04941105).

\section{PCSK9 and inflammation/thrombosis}

PCSK9 is a protein that is expressed in the liver, intestine, and kidneys, while circulating PCSK9 originates exclusively from hepatocytes $[16,17]$. The physiological role of PCSK9 is to

Address for correspondence: Przemysław Podhajski, MD, Department of Cardiology and Internal Medicine, University Hospital No. 1, ul. M. Skłodowskiej-Curie 9, 85-094 Bydgoszcz, Poland, tel: +4852 585 4023, fax: +4852585 4024, e-mail: przemo.podhajski@gmail.com

Received: 18.09.2021 Accepted: 19.10.2021 Early publication date: 15.11.2021

This article is available in open access under Creative Common Attribution-Non-Commercial-No Derivatives 4.0 International (CC BY-NC-ND 4.0) license, allowing to download articles and share them with others as long as they credit the authors and the publisher, but without permission to change them in any way or use them commercially. 
mediate the LDLR degradation and thus to regulate the LDL cholesterol (LDL-C) homeostasis [18]. Moreover, PCSK9 has also been shown to have a pro-inflammatory effect $[12,19,20]$. Overexpression of PCSK9 in macrophages increases the expression of IL- $1 \beta$ and TNF- $\alpha$, and decreases the anti-inflammatory markers Arg1 and IL-10 [21]. PCSK9 can act as trigger of inflammatory response as it causes increased expression of Lectin-like oxidized LDL receptor-1 (LOX-1) enhancing oxidized LDL (ox-LDL) uptake and amplifying the inflammatory response [10]. This pivotal interaction links cholesterol accumulation and chronic inflammatory process of atherosclerosis. Further data suggest that the nuclear factor kappa $\mathrm{B}(\mathrm{NF}-\kappa \mathrm{B})$ signaling pathway plays a key role in PCSK9-mediated vascular inflammation and thrombosis [20-22]. In fact, PCSK9 inhibition by using small interfering RNA (siRNA) has shown to suppress expression of LOX-1 and secretion of pro-inflammatory cytokines in macrophages by inhibiting $\mathrm{NF}-\kappa \mathrm{B}$ translocation into the nucleus [22-26].

\section{PCSK9 during infection}

Experimental data have shown that that heightened inflammation mediated by IL-6, may represent the biochemical link between HIV/HCV coinfection and elevated PCSK9 levels. This relationship may be bidirectional (PCSK9 regulating levels of IL-6) because healthy individuals with PCSK9 loss of function mutations have less IL-6 in response to lipopolysaccharide-induced inflammation [7, 27]. Dwivedi et al. [14] found that overexpression of PCSK9 was associated with increased liver and kidney pathology, plasma IL-6, alanine aminotransferase, and thrombin-antithrombin complexes concentrations during sepsis, whereas PCSK9 knockout mice exhibited reduced bacterial loads, lung and liver pathology, myeloperoxidase activity, plasma IL-10, and cell-free DNA (a procoagulant molecule released mainly by activated neutrophils) in a murine model of sepsis. Moreover, dyspnea, cyanosis, and overall grimace scores (severity of pain assessment) were higher in septic mice overexpressing PCSK9. Furthermore, lower expression of inflammation in PCSK9 knockout mice was confirmed by retained core body temperature during sepsis [14]. Results of this comprehensive experiment strongly suggest the strong impact of PCSK9 expression also on systemic, but not only local, inflammation and coagulation. These observations were in line with other data [27], which demonstrated that human PCSK9 loss-of-function genetic variants were associated with improved survival in septic shock patients and a decrease in systemic inflammatory cytokine response both in septic shock patients and in healthy volunteers after lipopolysaccharides (pathogenic lipid moieties from Gram-negative bacteria cell walls) administration. Moreover, a positive correlation between plasma levels of PCSK9 and TNF- $\alpha$, in a population of overall healthy subjects further supports the impact of PCSK9 on the systemic inflammatory response [28].

\section{PCSK9 inhibition and inflammation}

PCSK9 inhibition is associated with reduced monocyte recruitment and attenuated ox-LDL-induced expression of pro-inflammatory chemokine synthesis and secretion. Anti-PCSK9 antibodies alirocumab and evolocumab have been shown to decrease LDL-C level and reduce cardiovascular events in multiple clinical studies [29-36]. An experimental study in a mice model confirmed cholesterol lowering effect of PCSK9 inhibition and atherosclerosis development prevention also shows a reduction of inflammatory markers in mononuclear cells (IL-6, TNF- $\alpha$ mRNA) and in serum (CXCL-1, -10, -13, complement factor C5a) [16]. Furthermore, reduction of macrophage plaque infiltration and inflammation was found. These effects were associated with increased number of circulating endothelial progenitor cells and circulating angiogenic cells that are considered markers of endothelial and vascular health and are associated with positive clinical outcomes [15]. Additionally, reduced PCSK9 function is associated with increased pathogen lipid clearance via the LDLR, a decreased inflammatory response, and improved outcome in septic shock [27].

\section{Interleukin-6 in COVID-19 cytokine storm}

Infection with severe acute respiratory syndrome coronavirus-2 (SARS-CoV-2) has been classified into three clinical stages, regarding the severity and prognosis. Stage I is defined by mild unspecified symptoms of infection, such as myalgia, dry cough, headache, and subfebrile temperature, without any laboratory and radiological abnormalities. Stage II is characterized by cough, high fever, dyspnea, abnormal thoracic imaging, lymphopenia, and increased levels of inflammatory markers. This stage is further divided according to the presence (IIb) or absence (IIa) of hypoxemia. Stage III dis- 
plays clinical manifestations of a severe systemic inflammatory syndrome, culminating in severe respiratory failure with an unfavorable prognosis $[37,38]$. The increased levels of a large array of pro-inflammatory cytokines has been recognized as crucial in the pathophysiology of severe COVID-19 $[8,39]$.

Hyper-inflammatory response, also referred to as "cytokine storm" is an immune dysregulation which can lead to multiorgan failure and death through systemic inflammation. Various therapies, pathogens, cancers, autoimmune conditions, and monogenic disorders can trigger the immune hyperactivation related to unrestrained cytokine release [40]. Generally, the cytokine pattern depends on the underlying cause. The list of potential symptoms of cytokine storm significantly overlap with symptoms of COVID-19, and include high grade fever, fatigue, cough, headache, diarrhea, arthralgia, myalgia, disseminated intravascular coagulation dyspnea, hypoxemia, hypotension, vasodilatory shock, acute respiratory distress syndrome and multiple organ dysfunction syndrome [40, 41].

Cytokine storm with excessive release of inflammatory mediators induced by SARS-CoV-2 is a major cause of disease severity and death. In recent studies conducted in adults with polymerase chain reaction-proven SARS-CoV-2 infection IL-6 level was shown predict death better than age or $\mathrm{C}$-reactive protein (CRP). Moreover, the kinetic quantification of IL-6 levels allowed early discrimination between survivors and non-survivors [42]. These findings were in line with several other studies showing its good correlation to disease severity, the risk of needing mechanical ventilation, or death [43-47]. IL-6 has also been shown to be a prognostic marker of clinical worsening within 1-2 days from stage IIb to stage III. This pattern was not observed for CRP levels, despite the positive correlation between IL- 6 and CRP [42]. Hadjadj et al. [48] showed that IL-6 was not detected in peripheral blood at the transcriptional level, contrasting with high amounts of IL-6 protein during the inflammatory response in COVID-19. Expression of IL-6-induced genes (IL6R, SOCS3, and STAT3) was significantly increased, reflecting the activation of the IL-6 signaling pathway. TNF- $\alpha$ was only moderately up-regulated at the transcriptional level, whereas circulating TNF- $\alpha$ was significantly increased. Accordingly, TNF pathway-related genes were also up-regulated, including TNFSF10, which supports TNF- $\alpha$ having an important role in the induction of inflammation [43, 44, 48]. Inflammatory markers decline in patients who clinically improve (transition from phase IIb to IIa) and increase in those who experience worsening (transition from phase IIb to III). The critical inflammation point based on IL-6 monitoring of the disease seems to occur around 1 week to 10 days after the onset of symptoms [42]. This is in line with clinical observations in severe COVID-19 cases typically showing a two-step disease progression, starting with a mild-to-moderate presentation followed by a secondary respiratory worsening 9 to 12 days after the first onset of symptoms. This biphasic evolution marked by a substantial increase of acute phase reactants in the blood suggests a dysregulated inflammatory host response, resulting in an imbalance between pro- and anti-inflammatory mediators [48].

Therefore, it was hypothesized that there is a narrow period of time in which immunomodulatory drugs may be particularly effective and that therapeutics guided by the IL-6 level, in which randomization would occur only in patients with levels of IL-6 above a certain cut-off, could guide new therapeutic strategies and further improve outcomes [42].

Interleukin-6 repetitively emerges in subsequent reports from studies evaluating excessive cytokine release in patients with COVID-19 indicating a crucial role this cytokine plays in the pathophysiology of the disease. Many different cell types, e.g. monocytes, macrophages, fibroblasts, keratinocytes, astrocytes, endothelial cells, activated $B$ cells and T cells, can secrete IL- 6 upon appropriate stimulation [49, 50]. The main roles of IL-6 include regulation of cell responses of B and $\mathrm{T}$ cells and coordinates the activity of the innate and the adaptive immune systems [50].

\section{Clinical efficacy of selective inhibition of IL-6 in COVID-19}

Due to its central role in the cytokine storm during COVID-19, IL-6 signaling has been targeted as one of the most promising directions in the treatment of excessive inflammatory response in patients with SARS-CoV-2 infection [51]. Out of several anti-IL-6 pathway substances that are approved in various indications (Table 1), baricitinib, sarilumab and tocilizumab were evaluated in COVID-19.

The largest randomized clinical trial evaluating barcitinib, a Janus kinase (JAK) inhibitor, in a treatment of COVID-19 included 1033 patients. Barcitinib used together with remdesivir led to shorter time to recovery ( 7 vs. 8 days) and acceler- 
Table 1. Approved drugs that target interleukin-6 signaling.

\begin{tabular}{lcc}
\hline Compound & Target & Medical conditions \\
\hline Baricitinib & JAK-1, JAK-2 & Rheumatoid arthritis, atopic dermatitis \\
Filgotinib & JAK-1 & Rheumatoid arthritis \\
Ruxolitinib & JAK-1, JAK-2 & Myelofibrosis, polycythaemia vera \\
Sarilumab & IL-6R & Rheumatoid arthritis \\
Siltuximab & IL-6 & Castleman disease \\
Tocilizumab & IL-6R & Rheumatoid arthritis, juvenile idiopathic arthritis, Castleman \\
disease, giant cell arteritis, cytokine release syndrome & Rheumatoid arthritis, Psoriatic arthritis, ulcerative colitis \\
Tofacitinib & JAK-1, JAK-2, JAK-3 & Rheumatoid arthritis \\
Upadacitinib & JAK-1 &
\end{tabular}

ated improvement in clinical status (10 vs. 18 days) compared with patients receiving remdesivir alone. The 28-day mortality was numerically lower in the combined treatment group, but without reaching the statistical significance $(5.1 \%$ vs. $7.8 \%$, hazard ratio [HR] 0.65 ; 95\% confidence interval [CI] 0.39 -1.09 ) [52]. Meta-analyses of the efficacy of JAK inhibitors in COVID-19 suggest their impact on the reduced risk of mortality (odds ratio [OR] 0.51, $95 \%$ CI $0.28-0.93, \mathrm{p}=0.02$ ), with higher relative risk reduction observed with barcitinib compared with ruxolitinib, as well as clinical improvement (OR 1.76, 95\% CI 1.05-2.95, $\mathrm{p}=0.032$ ) [53, 54]. The main limitation of available data on barcitinib in COVID-19 is a lack of large, uniformly reported randomized studies.

Sarilumab is a monoclonal antibody that targets the IL-6 receptor (IL-6R). In a study by Lescure et al. [55] sarilumab compared with placebo did not reduce time to improvement or survival rate at 28 days in hospitalized patients with SARS-CoV-2 infection who required supplemental oxygen. On the other hand, in the REMAP-CAP study blockade of IL- $6 \mathrm{R}$ by sarilumab or tocilizumab compared with placebo resulted in improved in-hospital survival in critically ill COVID-19 patients $(78 \%, 72 \%$, and $64 \%$, respectively) [56]. However, clinical application of these results remains limited due to the fact that the sarilumab arm included only 48 patients.

Much more data is available on the use of tocilizumab for the treatment of patients with SARS-CoV-2 infection. Numerous trials with this IL-6R antagonist have been conducted so far, and meta-analyses of these heterogeneous studies indicate that use of tocilizumab is associated with decreased risk of death, but does not demonstrate benefits for surrogate endpoints including intensive care unit admission, invasive mechanical ventilation or secondary infections $[57,58]$.

\section{PCSK9 and thrombosis}

COVID-19 patients are at heightened risk of thrombosis [2-5]. PCSK9 might be implicated in the increased thrombotic risk during the more advanced stages of COVID-19.

Several mechanisms support this hypothesis. A recent investigation found that PCSK9 directly causes platelet aggregation by activating the CD36 downstream signalling pathways [14]. Increased plasma PCSK9 levels lead to elevated LDL and, subsequently, ox-LDL levels. The ox-LDL binds to the lecithin-like ox-LDL receptor (LOX-1) and CD36 on platelets and activates cytosolic phospholipase A2 (cPLA2). CD36 binds various ligands, leading to different effects. In particular, cPLA2 releases arachidonic acid from membrane phospholipids that is subsequently converted to thromboxane (Tx) A2 by cyclooxygenase (COX)-1/ /thromboxane synthase activity. TxA2 acts synergistically with downstream signalling generated by the binding of platelet agonists (adenosine diphosphate, collagen, and thrombin) to respective receptors to activate glycoprotein (GP) IIb/IIIa receptors. Activated GP IIb/IIIa receptors from adjacent platelets bind to fibrinogen forming platelet aggregation [23]. Increased plasma PCSK9 is associated with increased platelet activation in acute coronary syndrome [35].

Experimental data in the CD36-knockout mice model demonstrated that enhancing effects of PCSK9 on platelet activation are mediated by a direct binding on CD36 platelet surface and abolished by administration of PCSK9i or acetylsalicylic acid [14]. Notably, in the animal model of myocardial infarction, PCSK9-dependent platelet activation triggered microvascular obstruction and promoted the expansion of the infarction, possibly through increased oxidative stress, PCSK9 also modulates 


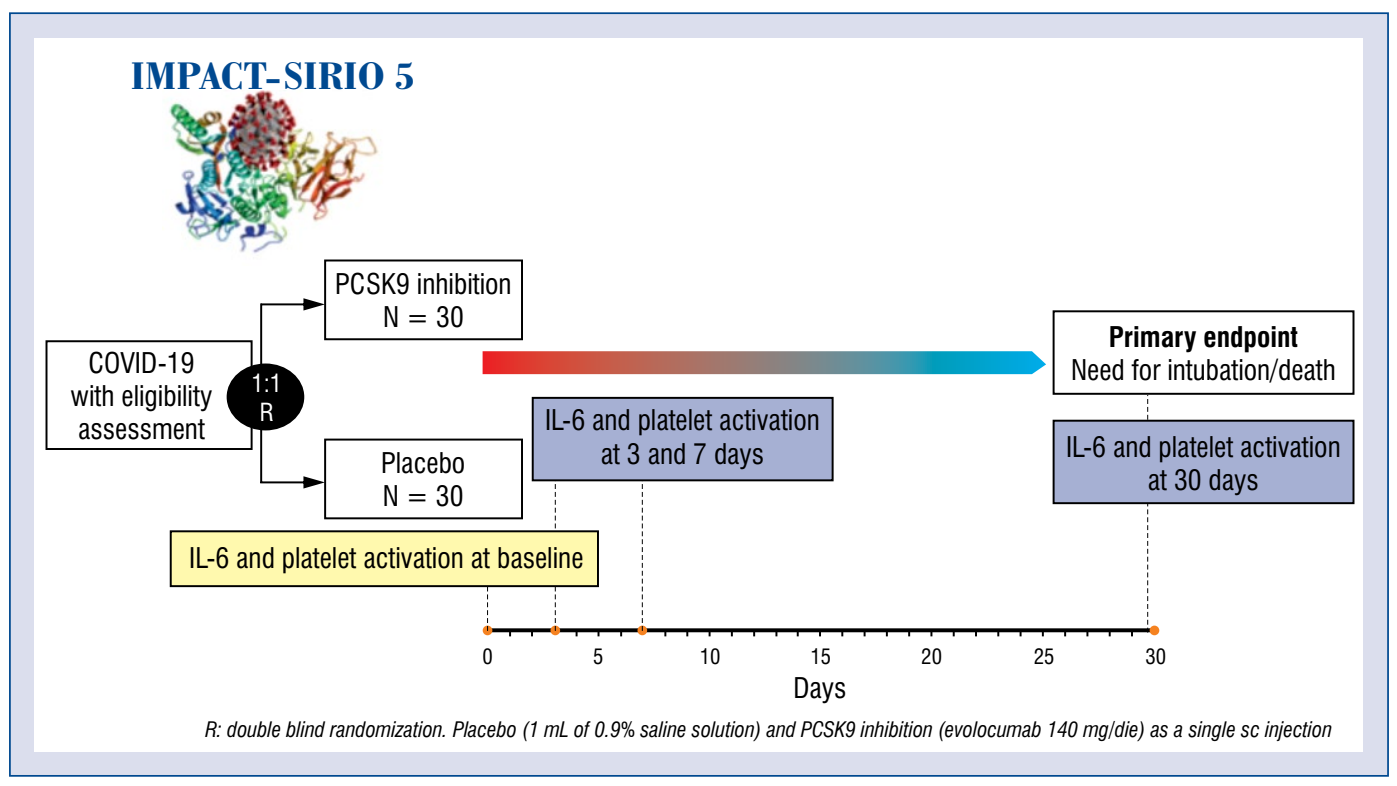

Figure 1. Proprotein convertase subtilisin/kexin type 9 (PCSK9) inhibition on clinical outcome in patients; COVID-19 — coronavirus disease 19; IL-6 — interleukin 6.

thrombosis by modifying platelet steady-state, leukocyte recruitment and clot formation [59]. In particular, platelets from PCSK9 knockout mice showed a significant reduction of glycoprotein $\mathrm{IIb} / \mathrm{III}$ and P-selectin expression, as well as of circulating platelet-leukocyte aggregates compared to wild type mice, indicating a lower platelet activation in the former [60].

\section{Methods}

Despite the progress in vaccination campaigns against SARS-CoV-2, the number of new variants and active infections continues to grow [61].

PCSK9 inhibition may represent a novel therapeutic pathway in COVID-19 which can act on top of those previously included into standard therapeutic approaches in COVID-19. The present study was designed to evaluate the impact of PCSK9 inhibition on clinical outcome in patients during the inflammatory stage of the SARS-CoV-2 infection.

This is a phase III, multicenter, double-blind, randomized, investigator-initiated clinical trial evaluating the efficacy and safety of PCSK9 inhibitors in the treatment of SARS-CoV-2 infected patients with cytokine storm stage. Patients will be randomized in 1:1 ratio into one of two study arms: treatment group and control group. $\mathrm{Pa}-$ tients assigned to the treatment group will receive a PCSK9 inhibitor evolocumab. Patients in the control group will receive an injection of $1 \mathrm{~mL}$ of $0.9 \%$
$\mathrm{NaCl}$. In addition, all people included in the study will be treated in accordance with the current therapeutic recommendations for COVID-19 patients. All study participants will be followed up for 30 days. In addition, a safety follow-up extended to 1 year is planned with an additional evaluation of functioning in disease with the Functioning in Chronic Diseases Scale (FCIS) [62-65]. The primary endpoint is the composite of need for intubation or all-cause death.

As there are no previous data to determine the number of subjects to be enrolled into the study for adequate power we have started a pilot study: Impact of PCSK9 inhibition on clinical outcome in patients during the inflammatory stage of the COVID-19 (IMPACT-SIRIO 5); ClinicalTrials. gov Identifier: NCT04941105. It was designed as the randomized, double-blind, multicenter, phase III study with a 30-day follow-up. The study was approved on October 27, 2020 by The Ethics Committee of Nicolaus Copernicus University in Torun, Collegium Medicum in Bydgoszcz (study approval reference number KB468/2020).

The plan is to enroll 60 consecutive patients in a 1:1 ratio to the experimental and control arm. In the experimental arm $140 \mathrm{mg}$ of evolocumab as a single subcutaneous injection is administered, while in the control arm $1 \mathrm{~mL}$ of $0.9 \%$ saline solution given as a single subcutaneous injection serves as a comparator. All patients are treated in accordance to the latest recommendations on caring for patients infected with SARS-CoV-2 (Fig. 1). 
Primary outcome measure

- Need for intubation (the indications for intubation determined individually for each patient and clinical status) or

- Death from any cause

\section{Secondary laboratory endpoint}

1. Change in serum IL-6 concentration from day 0 to day 7

2. Change in serum IL- 6 concentration from day 0 to day 30

\section{Secondary outcome measures}

1. The time of intubation

2. The time with non-invasive mechanical ventilation or high-flow nasal cannula

3. The time with oxygen therapy

4. The duration of hospitalization

5. Individual components of primary outcome

\section{Inclusion criteria}

1. Written informed consent for participation in the study

2. Male and female age $\geq 18$ at the time of signing the informed consent

3. SARS-CoV-2 infection confirmed by real-time reverse transcription polymerase chain reaction

4. COVID-19 pneumonia with typical radiological changes

5. $\mathrm{PaO}_{2} / \mathrm{FIO}_{2}$ ratio $\leq 300$

6. COVID-19 cytokine storm with elevated serum level of IL-6

\section{Exclusion criteria}

1. Use of fibrates other than fenofibrate or fenofibric acid

2. Known active infections or other clinical condition that contraindicate PCSK9 inhibitors

3. Known systemic hypersensitivity to PCSK9 inhibitors

4. Estimated glomerular filtration rate $<30 \mathrm{~mL} /$ $/ \mathrm{min} / 1.73 \mathrm{~m}^{2}$

5. Absolute neutrophil count $<2000 / \mathrm{mm}^{3}$

6. A platelet count $<50000 / \mathrm{mm}^{3}$

7. Creatine kinase greater than $3 \times$ upper limit of normal

8. Aspartate aminotransferase or alanine aminotransferase greater than $3 \times$ upper limit of normal

9. Not expected to survive for $>48$ hours from screening

10. Unrelated co-morbidity with life expectancy $<3$ months

11. Pregnancy
12. Any physical examination findings and/or history of any illness that, in the opinion of the study investigator, might confound the results of the study or pose an additional risk to the patient by their participation in the study

13. Patient being treated with other immunomodulators (except for glucocorticoids)

14. Patient included in any other interventional trial

\section{Discussion}

Summing up, strong scientific evidence exists that PCSK9 promotes the systemic inflammatory response. On the other hand, PCSK9 inhibition has been shown to reduce IL- 6 mediated inflammation pathway in experimental studies. Hyperinflammatory response, also called a "cytokine storm" with excessive release of inflammatory mediators induced by SARS-CoV-2 is a major cause of COVID-19 severity and death. IL-6 has been shown to be the best prognostic marker of clinical deterioration and mortality in patients developing cytokine storm. It was hypothesized that there is a narrow time window in which IL-6-guided immunomodulatory therapy may be particularly effective. Analysis of mortality by baseline IL- 6 values (above or below median) is planned. Moreover, according to available data, therapy with PCSK9 inhibitors is expected to reduce the rate of thrombotic complications due to the antiplatelet effect.

Conflict of interest: None declared

\section{References}

1. Xie Y, Wang Z, Liao H, et al. Epidemiologic, clinical, and laboratory findings of the COVID-19 in the current pandemic: systematic review and meta-analysis. BMC Infect Dis. 2020; 20(1): 640, doi: 10.1186/s12879-020-05371-2, indexed in Pubmed: 32867706.

2. Behrens EM, Koretzky GA. Review: cytokine storm syndrome: looking toward the precision medicine era. Arthritis Rheumatol. 2017; 69(6): 1135-1143, doi: 10.1002/art.40071, indexed in Pubmed: 28217930 .

3. Corradini E, Ventura P, Ageno W, et al. SIMI-COVID-19 Collaborators. Clinical factors associated with death in 3044 COVID-19 patients managed in internal medicine wards in Italy: results from the SIMI-COVID-19 study of the Italian Society of Internal Medicine (SIMI). Intern Emerg Med. 2021; 16(4): 1005-1015, doi: 10.1007/s11739-021-02742-8, indexed in Pubmed: 33893976.

4. Grasselli G, Greco M, Zanella A, et al. COVID-19 Lombardy ICU Network. Risk factors associated with mortality among patients with COVID-19 in intensive care units in lombardy, Italy. JAMA Intern Med. 2020; 180(10): 1345-1355, doi: 10.1001/jamainternmed.2020.3539, indexed in Pubmed: 32667669. 
5. Zangrillo A, Beretta L, Scandroglio AM, et al. COVID-BioB Study Group. Characteristics, treatment, outcomes and cause of death of invasively ventilated patients with COVID-19 ARDS in Milan, Italy. Crit Care Resusc. 2020; 22(3): 200-211, indexed in Pubmed: 32900326.

6. Yang X, Yu Y, Xu J, et al. Clinical course and outcomes of critically ill patients with SARS-CoV-2 pneumonia in Wuhan, China: a single-centered, retrospective, observational study. Lancet Respir Med. 2020; 8(5): 475-481, doi: 10.1016/s2213-2600(20)30079-5.

7. Mulchandani R, Lyngdoh T, Kakkar AK. Deciphering the COVID-19 cytokine storm: Systematic review and meta-analysis. Eur J Clin Invest. 2021; 51(1): e13429, doi: 10.1111/eci.13429, indexed in Pubmed: 33058143.

8. Del Valle DM, Kim-Schulze S, Huang HH, et al. An inflammatory cytokine signature predicts COVID-19 severity and survival. Nat Med. 2020; 26(10): 1636-1643, doi: 10.1038/s41591-020-1051-9, indexed in Pubmed: 32839624.

9. Mehta P, McAuley D, Brown M, et al. COVID-19: consider cytokine storm syndromes and immunosuppression. Lancet. 2020; 395(10229): 1033-1034, doi: 10.1016/s0140-6736(20)30628-0, indexed in Pubmed: 32192578.

10. Navarese EP, Kołodziejczak M, Dimitroulis D, et al. From proprotein convertase subtilisin/kexin type 9 to its inhibition: state-of-the-art and clinical implications. Eur Heart J Cardiovasc Pharmacother. 2016; 2(1): 44-53, doi: 10.1093/ehjcvp/pvv045, indexed in Pubmed: 27533061.

11. Mach F, Baigent C, Catapano AL, et al. ESC Scientific Document Group. 2019 ESC/EAS Guidelines for the management of dyslipidaemias: lipid modification to reduce cardiovascular risk. Eur Heart J. 2020; 41(1): 111-188, doi: 10.1093/eurheartj/ehz455, indexed in Pubmed: 31504418.

12. Ricci C, Ruscica M, Camera M, et al. PCSK9 induces a proinflammatory response in macrophages. Sci Rep. 2018; 8(1): 2267, doi: 10.1038/s41598-018-20425-x, indexed in Pubmed: 29396513.

13. Yang CL, Zeng YD, Hu ZX, et al. PCSK9 promotes the secretion of pro-inflammatory cytokines by macrophages to aggravate $\mathrm{H} / \mathrm{R}$-induced cardiomyocyte injury via activating NF- $\kappa \mathrm{B}$ signalling. Gen Physiol Biophys. 2020; 39(2): 123-134, doi: 10.4149/ gpb-2019057, indexed in Pubmed: 32329440.

14. Dwivedi DJ, Grin PM, Khan M, et al. Differential expression of PCSK9 modulates infection, inflammation, and coagulation in a murine model of sepsis. Shock. 2016; 46(6): 672-680, doi: 10.1097/SHK.0000000000000682, indexed in Pubmed: 27405064.

15. Schuster S, Rubil S, Endres M, et al. Anti-PCSK9 antibodies inhibit pro-atherogenic mechanisms in APOE*3Leiden.CETP mice. Sci Rep. 2019; 9(1): 11079, doi: 10.1038/s41598-01947242-0, indexed in Pubmed: 31366894.

16. Seidah NG, Benjannet S, Wickham L, et al. The secretory proprotein convertase neural apoptosis-regulated convertase 1 (NARC-1): liver regeneration and neuronal differentiation. Proc Natl Acad Sci U S A. 2003; 100(3): 928-933, doi: 10.1073/ pnas.0335507100, indexed in Pubmed: 12552133.

17. Zaid A, Roubtsova A, Essalmani R, et al. Proprotein convertase subtilisin/kexin type 9 (PCSK9): hepatocyte-specific low-density lipoprotein receptor degradation and critical role in mouse liver regeneration. Hepatology. 2008; 48(2): 646-654, doi: 10.1002/ hep.22354, indexed in Pubmed: 18666258.

18. Ferri N, Ruscica M. Proprotein convertase subtilisin/kexin type 9 (PCSK9) and metabolic syndrome: insights on insulin resistance, inflammation, and atherogenic dyslipidemia. Endocrine. 2016; 54(3): 588-601, doi: 10.1007/s12020-016-0939-0, indexed in Pubmed: 27038318.
19. dos Santos C, Marshall JC. Bridging lipid metabolism and innate host defense. Sci Transl Med. 2014; 6(258): 258fs41, doi: 10.1126/scitranslmed.3010501, indexed in Pubmed: 25320231.

20. Walley KR, Thain KR, Russell JA, et al. PCSK9 is a critical regulator of the innate immune response and septic shock outcome. Sci Transl Med. 2014; 6(258): 258ra143, doi: 10.1126/scitranslmed.3008782, indexed in Pubmed: 25320235.

21. Giunzioni I, Tavori H, Covarrubias R, et al. Local effects of human PCSK9 on the atherosclerotic lesion. J Pathol. 2016; 238(1): 52-62, doi: 10.1002/path.4630, indexed in Pubmed: 26333678.

22. Momtazi-Borojeni AA, Sabouri-Rad S, Gotto AM, et al. PCSK9 and inflammation: a review of experimental and clinical evidence. Eur Heart J Cardiovasc Pharmacother. 2019; 5(4): 237-245, doi: 10.1093/ehjcvp/pvz022, indexed in Pubmed: 31236571.

23. Gurbel PA, Navarese EP, Tantry US. Exploration of PCSK9 as a cardiovascular risk factor: is there a link to the platelet? J Am Coll Cardiol. 2017; 70(12): 1463-1466, doi: 10.1016/j. jacc.2017.07.779, indexed in Pubmed: 28911509.

24. Luquero A, Badimon L, Borrell-Pages M. PCSK9 functions in atherosclerosis are not limited to plasmatic LDL-cholesterol regulation. Front Cardiovasc Med. 2021; 8: 639727, doi: 10.3389/ fcvm.2021.639727, indexed in Pubmed: 33834043.

25. Qi Z, Hu L, Zhang J, et al. PCSK9 (proprotein convertase subtilisin/kexin 9) enhances platelet activation, thrombosis, and myocardial infarct expansion by binding to platelet CD36. Circulation. 2021; 143(1): 45-61, doi: 10.1161/CIRCULATIONAHA.120.046290, indexed in Pubmed: 32988222.

26. Tang $Z$, Jiang Lu, Peng J, et al. PCSK9 siRNA suppresses the inflammatory response induced by oxLDL through inhibition of NF- $\kappa$ B activation in THP-1-derived macrophages. Int J Mol Med. 2012; 30(4): 931-938, doi: 10.3892/ijmm.2012.1072, indexed in Pubmed: 22825241.

27. Walley KR, Thain KR, Russell JA, et al. PCSK9 is a critical regulator of the innate immune response and septic shock outcome. Sci Transl Med. 2014; 6(258): 258ra143, doi: 10.1126/scitranslmed.3008782, indexed in Pubmed: 25320235.

28. Ruscica M, Ferri N, Fogacci F, et al. Brisighella Heart Study Group. Circulating levels of proprotein convertase subtilisin/kexin type 9 and arterial stiffness in a large population sample: data from the brisighella heart study. J Am Heart Assoc. 2017; 6(5), doi: 10.1161/JAHA.117.005764, indexed in Pubmed: 28468788.

29. Bohula EA, Giugliano RP, Leiter LA, et al. Inflammatory and cholesterol risk in the FOURIER trial. Circulation. 2018; 138(2): 131-140, doi: 10.1161/CIRCULATIONAHA.118.034032, indexed in Pubmed: 29530884.

30. Robinson JG, Farnier M, Krempf M, et al. ODYSSEY LONG TERM Investigators. Efficacy and safety of alirocumab in reducing lipids and cardiovascular events. N Engl J Med. 2015; 372(16): 1489-1499, doi: 10.1056/NEJMoa1501031, indexed in Pubmed: 25773378.

31. Sabatine MS, Giugliano RP, Keech AC, et al. FOURIER Steering Committee and Investigators. Evolocumab and clinical outcomes in patients with cardiovascular disease. N Engl J Med. 2017; 376(18): 1713-1722, doi: 10.1056/NEJMoa1615664, indexed in Pubmed: 28304224.

32. Schwartz GG, Steg PG, Szarek M, et al. ODYSSEY OUTCOMES Committees and Investigators. Alirocumab and cardiovascular outcomes after acute coronary syndrome. N Engl J Med. 2018; 379(22): 2097-2107, doi: 10.1056/NEJMoa1801174, indexed in Pubmed: 30403574.

33. Navarese EP, Andreotti F, Raggi P, et al. Baseline low-density lipoprotein cholesterol to predict the extent of cardiovascular 
benefit from lipid-lowering therapies: a review. Eur Heart J Cardiovasc Pharmacother. 2019; 5(1): 47-54, doi: 10.1093/ehjcvp/ pvy038, indexed in Pubmed: 30247574.

34. Navarese EP, Kołodziejczak M, Petrescu A, et al. Role of proprotein convertase subtilisin/kexin type 9 inhibitors in patients with coronary artery disease undergoing percutaneous coronary intervention. Expert Rev Cardiovasc Ther. 2018; 16(6): 419-429, doi: 10.1080/14779072.2018.1474099, indexed in Pubmed: 29737886.

35. Navarese EP, Robinson JG, Kowalewski M, et al. Association between baseline LDL-C level and total and cardiovascular mortality after LDL-C lowering: a systematic review and meta-analysis. JAMA. 2018; 319(15): 1566-1579, doi: 10.1001/jama.2018.2525, indexed in Pubmed: 29677301.

36. Navarese EP, Kolodziejczak M, Schulze V, et al. Effects of proprotein convertase subtilisin/kexin type 9 antibodies in adults with hypercholesterolemia: a systematic review and meta-analysis. Ann Intern Med. 2015; 163(1): 40-51, doi: 10.7326/M14-2957, indexed in Pubmed: 25915661.

37. Siddiqi HK, Mehra MR. COVID-19 illness in native and immunosuppressed states: A clinical-therapeutic staging proposal. J Heart Lung Transplant. 2020; 39(5): 405-407, doi: 10.1016/j. healun.2020.03.012, indexed in Pubmed: 32362390.

38. Romagnoli S, Peris A, De Gaudio AR, et al. SARS-CoV-2 and COVID-19: From the Bench to the Bedside. Physiol Rev. 2020; 100(4): 1455-1466, doi: 10.1152/physrev.00020.2020, indexed in Pubmed: 32496872.

39. Huang $\mathrm{C}$, Wang $\mathrm{Y}, \mathrm{Li}$ X, et al. Clinical features of patients infected with 2019 novel coronavirus in Wuhan, China. Lancet. 2020; 395(10223): 497-506, doi: 10.1016/s0140-6736(20)30183-5.

40. Fajgenbaum DC, June CH. Cytokine storm. N Engl J Med. 2020; 383(23): 2255-2273, doi: 10.1056/NEJMra2026131, indexed in Pubmed: 33264547.

41. Gautret P, Million M, Jarrot PA, et al. Natural history of COVID-19 and therapeutic options. Expert Rev Clin Immunol. 2020; 16(12): 1159-1184, doi: 10.1080/1744666X.2021.1847640, indexed in Pubmed: 33356661.

42. Santa Cruz A, Mendes-Frias A, Oliveira AI, et al. Interleukin-6 is a biomarker for the development of fatal severe acute respiratory syndrome coronavirus 2 pneumonia. Front Immunol. 2021; 12: 613422, doi: 10.3389/fimmu.2021.613422, indexed in Pubmed: 33679753.

43. Laguna-Goya R, Utrero-Rico A, Talayero P, et al. IL-6-based mortality risk model for hospitalized patients with COVID-19. J Allergy Clin Immunol. 2020; 146(4): 799-807.e9, doi: 10.1016/j. jaci.2020.07.009, indexed in Pubmed: 32710975.

44. Aziz M, Fatima R, Assaly R. Elevated interleukin- 6 and severe COVID-19: A meta-analysis. J Med Virol. 2020; 92(11): 2283-2285, doi: 10.1002/jmv.25948, indexed in Pubmed: 32343429.

45. Grifoni E, Vannucchi V, Valoriani A, et al. Interleukin-6 added to CALL score better predicts the prognosis of COVID-19 patients. Intern Med J. 2021; 51(1): 146-147, doi: 10.1111/imj.14974, indexed in Pubmed: 33336833.

46. Herold T, Jurinovic V, Arnreich C, et al. Elevated levels of IL-6 and CRP predict the need for mechanical ventilation in COVID-19. J Allergy Clin Immunol. 2020; 146(1): 128-136.e4, doi: 10.1016/j. jaci.2020.05.008, indexed in Pubmed: 32425269.

47. Liu T, Zhang J, Yang Y, et al. The role of interleukin- 6 in monitoring severe case of coronavirus disease 2019. EMBO Mol Med. 2020; 12(7): e12421, doi: 10.15252/emmm.202012421, indexed in Pubmed: 32428990.

48. Hadjadj J, Yatim N, Barnabei L, et al. Impaired type I interferon activity and inflammatory responses in severe COVID-19 patients. Science. 2020; 369(6504): 718-724, doi: 10.1126/science. abc6027, indexed in Pubmed: 32661059.
49. Garbers C, Heink S, Korn T, et al. Interleukin-6: designing specific therapeutics for a complex cytokine. Nat Rev Drug Discov. 2018; 17(6): 395-412, doi: 10.1038/nrd.2018.45, indexed in Pubmed: 29725131.

50. Scheller J, Chalaris A, Schmidt-Arras D, et al. The pro- and anti-inflammatory properties of the cytokine interleukin-6. Biochim Biophys Acta. 2011; 1813(5): 878-888, doi: 10.1016/j. bbamcr.2011.01.034, indexed in Pubmed: 21296109.

51. Angriman F, Ferreyro B, Burry L, et al. Interleukin-6 receptor blockade in patients with COVID-19: placing clinical trials into context. Lancet Respir Med. 2021; 9(6): 655-664, doi: 10.1016/ s2213-2600(21)00139-9.

52. Kalil AC, Patterson TF, Mehta AK, et al. ACTT-2 Study Group Members. Baricitinib plus remdesivir for hospitalized adults with Covid-19. N Engl J Med. 2021; 384(9): 795-807, doi: 10.1056/ NEJMoa2031994, indexed in Pubmed: 33306283.

53. Wijaya I, Andhika R, Huang I, et al. The use of Janus Kinase inhibitors in hospitalized patients with COVID-19: Systematic review and meta-analysis. Clin Epidemiol Glob Health. 2021; 11: 100755, doi: 10.1016/j.cegh.2021.100755, indexed in Pubmed: 33969237.

54. Chen CX, Wang JJ, Li H, et al. JAK-inhibitors for coronavirus disease-2019 (COVID-19): a meta-analysis. Leukemia. 2021; 35(9): 2616-2620, doi: 10.1038/s41375-021-01266-6, indexed in Pubmed: 33990684.

55. Lescure FX, Honda H, Fowler R, et al. Sarilumab in patients admitted to hospital with severe or critical COVID-19: a randomised, double-blind, placebo-controlled, phase 3 trial. Lancet Resp Med. 2021; 9(5): 522-532, doi: 10.1016/s2213-2600(21)00099-0.

56. Gordon AC, Mouncey PR, Al-Beidh F, et al. REMAP-CAP Investigators. Interleukin-6 receptor antagonists in critically ill patients with COVID-19. N Engl J Med. 2021; 384(16): 1491-1502, doi: 10.1056/NEJMoa2100433, indexed in Pubmed: 33631065.

57. Khan FA, Stewart I, Fabbri L, et al. Systematic review and meta-analysis of anakinra, sarilumab, siltuximab and tocilizumab for COVID-19. Thorax. 2021; 76(9): 907-919, doi: 10.1136/thoraxjnl-2020-215266, indexed in Pubmed: 33579777.

58. Chen CX, Hu F, Wei J, et al. Systematic review and meta-analysis of tocilizumab in persons with coronavirus disease-2019 (COVID-19). Leukemia. 2021; 35(6): 1661-1670, doi: 10.1038/ s41375-021-01264-8, indexed in Pubmed: 34002026.

59. Luquero A, Badimon L, Borrell-Pages M. PCSK9 functions in atherosclerosis are not limited to plasmatic LDL-cholesterol regulation. Front Cardiovasc Med. 2021; 8: 639727, doi: 10.3389/ fcvm.2021.639727, indexed in Pubmed: 33834043.

60. Qi Z, Hu L, Zhang J, et al. PCSK9 (proprotein convertase subtilisin/kexin 9) enhances platelet activation, thrombosis, and myocardial infarct expansion by binding to platelet CD36. Circulation. 2021; 143(1): 45-61, doi: 10.1161/CIRCULATIONAHA.120.046290, indexed in Pubmed: 32988222.

61. www worldometers info. (accessed 2021).

62. Kubica A. Self-reported questionnaires for a comprehensive assessment of patients after acute coronary syndrome. Med Res J. 2019; 4(2): 106-109, doi: 10.5603/mrj.a2019.0021.

63. Michalski P, Kasprzak M, Kosobucka A, et al. Sociodemographic and clinical determinants of the functioning of patients with coronary artery disease. Med Res J. 2021; 6(1): 21-27, doi: 10.5603/ mrj.a2021.0003.

64. Buszko K, Pietrzykowski $€$, Michalski P, et al. Validation of the Functioning in Chronic Illness Scale (FCIS). Med Res J. 2018; 3(2): 63-69, doi: 10.5603/mrj.2018.0011.

65. Kubica A, Kosobucka A, Michalski P, et al. Self-reported questionnaires for assessment adherence to treatment in patients with cardiovascular diseases. Med Res J. 2018; 2(4): 115-122, doi: 10.5603/mrj.2017.0015. 